\title{
Integration of Smart Wearable Devices and Cloud Computing in the Kenyan Public Health Care System
}

\author{
Esther Nanzayi Ngayua * \\ Business School, University of Shanghai for Science and Technology, 516 Jungong Road, Shanghai \\ 200093,China \\ *E-mail of the corresponding author: esthernanzayi@yahoo.com
}

\begin{abstract}
The utilization of smart wearable devices and cloud computing in the Kenyan public health care system will facilitate real-time patient monitoring and management. The shortage of certified healthcare professionals and the limited access to quality specialized care for individuals in remote settings has prompted the adoption of wearable devices and cloud computing strategies in Kenya. However, there lacks a clear framework design of integrating the technologies in the public health sector. This article evaluates the current status of healthcare systems in Kenya. It also investigates the existing mobile health and cloud computing services in the country while evaluating the main legal concerns inherent to the utilization of the technologies. The document further outlines a framework design for a mobile application named GB Health. The application incorporates cloud computing and smart wearable devices in the Kenyan public health care system. The design will enhance workflow and patient outcomes in the sector.
\end{abstract}

Keywords: Smart wearable devices, cloud computing, GB Health

DOI: $10.7176 / \mathrm{IKM} / 11-4-04$

Publication date:June $30^{\text {th }} 2021$

\section{Introduction}

Health informatics is a combination of resources applied in the acquisition, storage, and retrieval of data to improve patient safety and quality of healthcare. The advent and integration of information technology systems has facilitated the identification of vulnerable individuals, which informs the emergency preparedness of the health care providers (Shah, Newell and Whitworth, 2016). Electronic health records provide a platform for collecting, analyzing, storing, and presenting healthcare information. However, there is a need for more secure and interoperable information systems that allow for real-time patient monitoring.

Wearable devices and cloud computing provide an interconnected system for continuous management of ill patients. The technologies apply the concepts of the Internet of Things (IoT) including the functionality and processing capabilities of the devices, integration with biosensors, and access to the Internet (Metcalf et al., 2016). Smart wearable devices collect vital data from the users through body sensors. The information is channeled via Internet connections to the physicians through portable devices, such as smartphones (Gupta, Chakraborty and Gupta, 2019). This allows for prompt identification of the patients' needs and automated interventions to ensure the wellbeing of the users.

Adoption of wearable devices in Kenya will increase the accessibility to quality healthcare, especially for individuals living in distant areas. It will also minimize medical costs for such individuals by remotely availing medical care to the users (mHealth Kenya Ltd, 2015). Moreover, the incidence of non-communicable diseases has been increasing in recent years. Such illnesses include hypertension, diabetes, and cancer (Freiberg et al., 2016). Advanced health informatics tools can be used in promoting preventive care services such as encouraging body exercises to curb the burden of lifestyle diseases.

The utilization of wearable devices will lead to the generation of large volumes of healthcare data. Cloud computing provides an efficient platform for the archiving and retrieval of healthcare information (Gupta, Chakraborty and Gupta, 2019). This will enhance care coordination between professionals and hence, improving the healthcare provision in the public sector. It also results in increased patient involvement and thus, personalized care (Hopia et al., 2015). This boosts individual health responsibility and establishment of customer-centric medical guidelines.

The article aims at establishing the current status of healthcare systems in Kenya. It also investigates the existing 
mobile health services in Kenya and the integration of wearable devices and cloud computing in the public health sector. The document further outlines a framework design for a mobile application named GB health. The application incorporates smart wearable devices and cloud computing in the Kenyan public health care system.

\section{Current State of the Kenyan Health Care System}

In Kenya, the health care system comprises of three sectors. These include the public health sector, the private sectors, and the faith-based organizations. The private sector is made up of for-profit organizations, such as private hospitals and over 6,000 local and international non-governmental organizations (mHealth Kenya Ltd, 2015). The public health sector makes up a larger portion of the healthcare system due to the high number of governmental facilities. The hospitals are structured into six levels. Specialized care is offered at the level six facilities and some level five hospitals. Initially, advanced medical care resources were centralized within the nation's capital, Nairobi (Davis et al., 2019). This made timely access to such services by individuals in rural and remote settings difficult.

The public healthcare sector in Kenya is strained owing to inadequate staffing and resource allocation. The government of Kenya spends approximately 6.7\% of GDP on public healthcare sector (Obadha et al., 2019). Also, the decline in funding over the years has led to increased out-of-pocket spending by the population, which accounts for $36.7 \%$ of the health sector revenue (Appleford and Ramarao, 2019). However, a majority of Kenyans are from low-income settings. As a result, access to medical care is reduced for the poor.

The private sector offers higher quality and more comprehensive services than public institutions. The private institutions have adequate staffing and availability of hi-tech resources. However, the cost of services in such institutions is high, accommodating only the middle- and high-income earners. The low-income population is unable to afford private medical care due to high drug prices and transport challenges (Kabia et al., 2019). Consequently, over-utilization of the public facilities occurs, which leads to depletion of the limited resources in such institutions.

The government through the Ministry of Health $(\mathrm{MOH})$, has adopted a strategic plan to improve the country's health care sector. These include the devolution of healthcare, expansion of the National Health Insurance Fund coverage, and increased foreign investment in health (mHealth Kenya Ltd, 2015). There has also been an increased construction of new hospitals to ease the burden on the already existing facilities. However, poor management, inadequate resource allocation, and lack of infrastructure continue to lower the care quality in the public health sector.

Also, a majority of the hospitals in Kenya use manual health records to store patient information. During the first visit, a patient file is created using demographics and case details. The file is circulated to the clinics visited by the patient for reference and addition of new information. Hospital cards are given to the patient to facilitate quicker identification of the patient's file. Over time, the process has proved tedious, time-consuming, expensive, unreliable, and inefficient (Fielding, Odero and Ochieng, 2016) This has prompted the adoption of automated registry processes, such as the Electronic Health Records (EHRs).

\section{Characteristics of Mobile Health Devices}

Mobile health (mHealth) platforms provide an avenue for engaging, educating, and empowering patients while enhancing workflow within a health system. This ultimately leads to the development of value-based care models, aimed at improving patient outcomes (Hamine et al., 2015). mHealth devices are wirelessly integrated with IoT servers allowing, real-time communication between caregivers and patients regardless of location on different platforms (Erdeniz et al., 2018). The main features of mHealth devices are discussed below.

\section{Portability}

Mobile-health devices are compact and easy to carry around, for example, smartphones like iPhones. Others include smartwatches, Personal Digital Assistants (PDA), portable media devices such as MP3/MP4 players, and ultra-portable computers, including tablets and laptops. These devices are light and can be carried around with ease in pockets worn in specific body parts. An example is the TICKER heart monitor by Wahoo Fitness, worn on the chest to check heart rate (Hettiarachchi et al., 2019). Portable mHealth devices allow for real-time delivery of interventions within the relevant context, such as a reminder to take drugs or go for an appointment.

The strategy is essential in providing easy access to clinical management support in situations or settings with 
limited availability of specialized health care. They also integrate with EHRs and IoT, allowing caregivers to remotely monitor the health indices of patients in different areas (Toma, Masuda and Yamamoto, 2019). This facilitates timely identification of disease outbreaks, fast provision of intervention measures, and strategic resource allocation. It also minimizes transcription errors in data collection.

\section{IP Enabled and Wireless Connectivity}

Each IoT enabled mHealth device has a designated Internet Protocol (IP) address. The identifier allows for geographical mapping of the devices while allowing the transfer and receipt of specific information within a network. It also facilitates search engine optimization for particular devices when using the Internet (Halepoto et al., 2018). IP works over wired communications such as Ethernet and wireless connections, including WIFI, 4G, $3 \mathrm{G}$ and LTE. Integration of IP with IoT in mHealth devices streamlines the billing, monitoring, diagnostic, and management processes (Plageras et al., 2016). The Internet connectivity of mHealth devices is guided by the power capacity, cost, software design, and connection speed.

\section{Low Power Consumption}

Mobile health devices are designed to have long-lasting battery capacities. The designs incorporate strategies such as power-saving modes (Cruz and Abreu, 2019). These are useful in scheduling measurement times to avoid unnecessary battery dissipation. They also apply hardware modifications such as the performance of specific tasks by the sensors and processors during periods of optimum charge only. Other features include the use of advanced biosensors, ultra-low power signaling, and screening detectors to ensure high precision measurements (Magno et al., 2017). These strategies minimize power usage and hence, prolonging the battery life of mHealth devices.

Some IoT systems utilized by the mHealth devices have a high energy conversion rate. This results from powerintensive processes such as frequent collection and processing of vast data. Materials such as piezoelectric and thermoelectric elements are incorporated into the devices' electronic systems to minimize dependence on the battery capacity (Din and Paul, 2019). The elements convert environmental components such as solar and vibration energy to usable power. Such modifications enhance the energy efficiency of the portable devices, promoting their utility.

\section{Existing Methods Used to Integrate Mobile Wearable Devices and Cloud Computing in Health Care}

The ratio of certified caregivers to the population density in Kenya is below the recommended international standards. This is attributed to brain drain, whereby trained professionals leave the country in search of better opportunities abroad (Dohlman et al., 2019). Healthcare systems are adopting devices that enhance the Internet of Things, such as mobile wearable devices, mHealth applications, and cloud computing to bridge the gap. In Kenya, approximately 45 mHealth projects have been launched(mHealth Kenya Ltd, 2015). The projects integrate concepts of Information and Communication Technology (ICT) and eHealth. The integration of such technologies in health increases access to timely and quality medical care for individuals in remote settings or emergencies.

The most common mobile wearable devices already adopted in Kenya are smartphones and smartwatches. According to a study by the Communications Authority of Kenya (2018) indicated that $87 \%$ of the total population are active mobile users. This is attributed to the market bulge of affordable gadgets (Perlman and Wechsler, 2019). The upsurge has created an opportunity for harnessing the power of technology in improving healthcare delivery.

In Kenya, a majority of the mobile phone subscribers use the less advanced gadgets that provide essential services such as messaging and calls. Healthcare providers utilize these platforms to send health-relevant messages to the target individuals through Short Messaging Services (SMS) and periodic alerts (Le-Tien et al., 2018). Initiatives that apply this strategy include the WelTel program that utilizes SMS to promote adherence to Anti-Retroviral Therapy among people living with HIV (Mills and Lester, 2019). The outcomes of the intervention include lowered healthcare costs due to suppression of viral loads.

Apart from the WelTel initiative, other public mobile health programs in Kenya include the Integrated Disease Surveillance Response that monitors disease occurrence in remote settings. The Clinton Health Access Initiative that focuses on increasing accessibility to mHealth technologies for individuals affected by HIV (Lam et al., 2019). The AMREF Jamii Smart initiative aims at developing mobile health devices and applications to improve 
maternal and child healthcare. Also, different companies are joining the mHealth market to offer practical solutions in the integration of mobile health devices in the Kenyan healthcare system. These include Safaricom, IntelliSOFT Consulting, Roamtech Solutions, and Fintech Group(mHealth Kenya Ltd, 2015). The companies provide software solutions that support healthcare access and delivery in the country. --

According to the mHealth strategic plan of 2015-2020, threats to the entry of mobile health devices in the public healthcare system include lack of expertise in the field. Also, there is a gap in the development of strong business partnerships between $\mathrm{MOH}$ and product vendors. Low supplier power owing to the increased competition within the ICT industry and the threat of cheaper substitute products also limits entry into the market (Munguti, 2018). The biggest buyers of the mHealth technologies in Kenya are healthcare donors and MOH. A majority of the products focus on acute and life-threatening conditions, as well as preventable illnesses such as Tuberculosis, HIV/AIDS, and malaria.

Cloud computing in Kenya is still in the developing stages. Adoption of cloud storage systems requires a high Internet speed connectivity, which can be costly to the healthcare institutions (Gill and Buyya, 2019). Some network company titans such as Safaricom and Seven Seas Technologies have introduced high speed fiber connectivity. This will facilitate growth of the cloud computing industry in Kenya (Kshetri and Alcantara, 2016). Also, healthcare institutions are employing informatics experts to provide oversight and direction in the adoption of cloud storage within the organizations.

The public healthcare system is yet to adopt cloud computing owing to the high initial cost, shortage of infrastructure, limited expertise knowledge, and the unwillingness of the care providers in governmental institutions (Munguti, 2018). Cloud providers targeting the Kenyan healthcare sector include Microsoft Cloud, Safaricom, MTN, and Cloudpap(mHealth Kenya Ltd, 2015). The companies are empowering the healthcare stakeholders to embrace the IoT of cloud computing through improved detection, diagnosis, treatment, and prevention of diseases within and without the clinical environment.

\section{Main Legal Considerations on Cloud Computing in Kenya}

In Kenya, the provisions in cybersecurity laws and regulations govern the adoption and utilization of cloud computing and IoT by the healthcare systems. International laws are also applied in addressing specific matters related to the use of cloud storage and IoT enabled devices in the country (Boopathy and Sundaresan, 2016). The main legal concerns inherent to cloud adoption and utility include data privacy and security concerns, liabilities, the rights and obligations of the involved parties, and procurement of infrastructure.

Data privacy and security issues in cloud computing include hacking, phishing, the introduction of malware, identity fraud, electronic theft, and unauthorized disclosure of access codes and security passwords (Alhenaki et al., 2019). It is imperative that the institutions uphold patient confidentiality in the management of healthcare data. The cloud computing users should critically assess the criteria used by the product vendors in ensuring protection and regulated access to the stored data.

In Kenya, the Computer Misuse and Cybercrimes Act of 2018 applies to the identification, prevention, and response to cyber and computer-related crimes. However, the Act does not provide penalties for any offences committed (Kimani, 2019). The Kenya Criminal Procedure Code is thus applied in the arraignment and sentencing of accused individuals. Provisions in the General Data Protection Regulations (GDPR) of the European Union are also applied. These include reporting of security breaches in the cloud to the regulatory authorities within seventy-two hours of occurrence (Voigt et al., 2017). It also stipulates the notification of the data owners in such situations and institution of security levels within the cloud.

Liability issues in cloud computing and IoT include ownership of intellectual property, contract terms, and occurrence of undesired situations such as data theft. Specific issues include interruption of information processes that result in downtime and potential financial loss for the users, during system updates and maintenance (Rahi, Bisui and Misra, 2017). Also, non-compliance with the principles of data integrity and protection, as well as negligence of the rights and obligations involved parties can be a liability for the product vendors and users.

In Kenya, the Health Act of 2017, article number 21, provides that an individual's consent must be obtained prior to provision of any health care service. It should also be sought before dissemination of personal information, in this case, storage of personal data in the cloud (Bitta et al., 2017). The MOH is also expected to protect, respect, and promote the rights of the patients to the highest attainable standards. The Act also stipulates that $\mathrm{MOH}$ oversees the adoption and maintenance of a comprehensive integrated healthcare information systems within the 
public health sector (mHealth Kenya Ltd, 2015). This ensures data safety while providing a regulatory framework for the institutions to minimize liability issues.

The rights of the data subjects include the ability to seek access to or erase their information from the cloud. They have a right to secure and clear information that they can understand. They also can withdraw consent at any given time (Bolognini and Balboni, 2019). Institutions that are using cloud computing have should uphold the rights of their clients. They are obligated to seek assessments and certification from regulatory bodies through legal processes (Boopathy and Sundaresan, 2016). They should also ensure compliance with the local and international guidelines of cloud computing and IoT, including the International Standards Organization requirements.

The Health Act of 2017 provides that purchase of health technologies be conducted in accordance with the Public Procurement and Disposal Act through the Kenya Medical Supplies Authority (KEMSA). However, the national government regulates the processes of procurement, distribution, and management of the resources (Barasa et al., 2017). The agency is in charge of providing health supplies directly to county referral hospitals.

\section{Methodology}

The author has visualized a framework design that integrates mobile wearable devices with the cloud in the Kenyan public health system. The architecture will enable caregivers to monitor and manage a range of patients consecutively and to improve public health outcomes (Alexandru et al., 2016). A mobile health application named GB health (an acronym for General Body Health) has been proposed. The app will be compatible with both android and the iPhone operating systems. It will be accessed and downloaded for free from the Google Play Store and the apple store.

\subsection{Design and Development of the Application}

The suggested health application will be designed in a manner that will allow integration into cellular gadgets that are enabled with Wireless body area networks (WBANs), General Packet Radio Service (GPRS), and the Global Positioning System (GPS). WBANs and GPRS will enable the transmission of data over wireless Internet connections, such as WIFI, and cellular networks (Bhardwaj and Sharma, 2018). They link to a linked electronic medical record of a chosen hospital.

The data will be stored in the individual's medical records in the institution and will then be uploaded to the cloud periodically. The public health institutions will have shared clouds that are linked to the patients' mobile devices to reduce costs. The facilities will share infrastructure and maintenance costs minimizing the expenditure on a single facility (Manya, Nielsen and Pundo, 2016). The application will be particularly helpful in situations where the user is far from their first-choice hospital. It will allow accessibility of patient data from other hospitals that share the cloud storage.

The GB Health application will be integrated with an SOS alert system that will be useful in prompt identification of emergencies. The medical team that has received the alert can then use GPS to track the individual and avail help at their specific location (Akram et al., 2017). The application interface will be userfriendly and easy to navigate. The application will have five sections, whose components and functions are described below. A visual representation of the GB Health app is shown in Figure 1. The navigation interface is shown in Figure 2 below. 


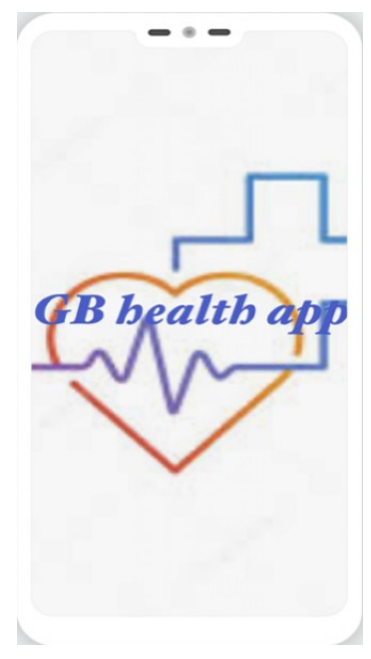

Figure 1: GB Health App

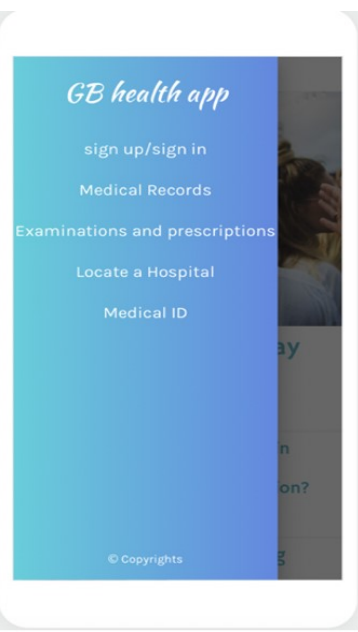

Figure 2: GB Health App Navigation Interface

\subsubsection{Sign-up}

This section will be used to create a personal profile and link the application to the wearable devices utilized by the user. A sign-up procedure will be used to obtain the user's identity and permission to ensure data safety (Taylor et al., 2018). In the sign-up section, basic personal information will be required, including the full name, sex, date of birth, identification number, and email address. In some cases, the user may also provide a profile picture. The section will also outline the terms and conditions of using the application, which the user needs to understand before agreeing to the privacy terms (Thinakaran et al., 2018). The user will be required to provide details of their wearable devices to link the application to the gadgets.

The user can only link devices registered to their names with the application. Also, the app will be designed in such a way as to restrict utility of a single account per mobile phone. The installation and launching process will have various security checks to ensure user authenticity (Costa et al., 2018). The user will also be required to specify a health institution where their data can be stored in the electronic health records before being transferred to the cloud.

\subsubsection{Medical Records}

This section will be divided into two parts; the past medical history data and the periodically recorded data. The section on periodically recorded data will contain information being uploaded to the application from the user's wearable device. This part will enable the user to access their recent medical data, including the last measurements of heart rate, body temperature, and blood pressure, among others. After a few hours, the periodically recorded data will then be transferred to the medical history section.

The section on the past medical history will contain information of on the previously used drugs, results of tests done, and an outline of previous readings collected by the wearable devices. The user will be required to key in specific data such as the laboratory tests done, the procedures undergone and the drugs taken. The data will then be sent to EHRs in the user's hospital of choice for clinician review before being uploaded to the cloud (Slotwiner et al., 2019). Depending on the information transferred to the caregivers, the healthcare providers can determine the medical state of the user and respond accordingly. Also, the section will allow access to the patient's previous medical history by different caregivers to facilitate care coordination, especially during emergency cases.

\subsubsection{Examinations and Prescriptions}

In this section, there will be three parts, including the consultation, prescription, and billing interfaces. In the consultation, the user will be able to communicate with a certified physician. The section will outline a list and brief description of the available doctors, from which the user can pick a doctor of their choice. The consultation 
can be through feedback messages or face-to-face communications. Upon consultation, the user will be directed to the prescriptions section. In this part, the user is able to access a list of the prescribed drugs, which they can order online or purchase at a pharmacy. The user is then directed to the billing section where they pay for the services offered via mobile money platforms or credit cards.

The section will require the user to fill out some details as part of a security check. These include names of their preferred doctors, credit card numbers, mobile phone numbers, among others. Verification codes will be used to ensure the authenticity of the user (Costa et al., 2018). This will be essential in maintaining patient privacy and ensuring the security of the users' funds.

\subsubsection{Locate a Hospital}

In this section, the user can access a geographical map to enable them to identify hospital institutions and pharmacies within their location radius. The section will utilize the Global Positioning System (GPS) to determine the user's current location and provide a direction to the chosen institution (Kumar et al., 2016). The section will also provide a link to Google Maps or Apple Maps for further searches. It will also give a time approximation of the duration taken to get to a chosen facility using different means of transport. Moreover, it will outline a brief description of the chosen facilities, including the operating hours, services offered, contact numbers, and the star ratings of the institution. A history of the previously selected facilities will be kept for quicker searches.

\subsubsection{Medical ID}

In this section, the user is allowed to store their individual medical information such as hospital number, emergency contacts, date of the next appointment, personal doctor's address, health insurance number, next of kin, nutrition and diet restrictions, drug allergies, and any other medical information deemed necessary.

\subsection{The Complexity of the Application}

Generally, the application will be user friendly and easy to navigate. Advertisements will be restricted to facilitate smooth user experience and avoid confusions. However, the level of complexity across the various sections of the application will be varied. The sign-up interface will have a low complexity as a step-by-step procedure will be used in obtaining the users' information. A high level of complexity will be encountered in the medical records section. This will be useful in restricting access or deletion of patient data from the application (Martínez-Pérez, de la Torre-Díez and López-Coronado, 2015). This will protect the integrity of the data in the hospital and cloud records.

The complexity of the examinations and prescription section will be medium. However, the billing and payment sub-section will be more complex than the consultation and prescription subsections to ensure the security of the patient's funds. Complexity in this section will arise from the need to fill in some personal information such as the national identification number, credit card number, and phone number. Also, verification codes will be used to ensure user authenticity.

The sections on locating a hospital and medical ID will also have a medium level of complexity. This will occur as a result of the requirement to fill out some details such as current location, emergency contacts, user's nutrition and drug specifications, among others. The complexity will be beneficial in restricting access and adulteration of the patient's records by third parties.

Table 1: Summary of the GB Health App

\begin{tabular}{|l|l|l|}
\hline Section & Description & Complexity \\
\hline Sign-up & $\begin{array}{l}\text { Creation of a GB Health individual } \\
\text { profile }\end{array}$ & Low \\
\hline Medical Records & Contains the user's medical history & High \\
\hline Examination and Prescription & $\begin{array}{l}\text { Allows the user to consult a certified } \\
\text { caregiver, obtain prescriptions, and } \\
\text { pay for the services rendered by the } \\
\text { physician. }\end{array}$ & Medium \\
\hline Locate a Hospital & $\begin{array}{l}\text { Uses GPS tracking to identify nearby } \\
\text { medical facilities. }\end{array}$ & Medium \\
\hline Medical ID & $\begin{array}{l}\text { Contains additional personal medical } \\
\text { data, such as emergency contacts. }\end{array}$ & Medium \\
\hline
\end{tabular}




\section{Conclusion}

In conclusion, mobile wearable devices and cloud computing are yet to be fully integrated into the Kenyan public health care system. The Ministry of Health is partnering with different organizations to incorporate mobile-health and cloud storage in care delivery. The adoption and utilization of cloud computing and wearable devices in Kenya is regulated by the Computer Misuse and Cybercrimes Act of 2018 and the Health Act of 2017. However, the incorporation of advanced technology in care delivery has concerns on data safety, liabilities, and the constitutional rights of the users and providers. The GB Health application offers a practical solution in the integration of mobile wearable devices and cloud computing in the public health care sector.

\section{Acknowledgements}

Special thanks also go to Dr. WeeMeng Yeo and Elem Miranda, Adam Smith School of Business, University of Glasgow, Dr. Yiquan Gu, Management School, University of Liverpool for their support.

\section{Conflict of Interest}

The authors state that there is no conflict of interest in this paper.

\section{References}

Akram, A. et al. (2017) 'Life savior: An integrated emergency response system', in ICIT 2017 - 8th International Conference on Information Technology, Proceedings. doi: 10.1109/ICITECH.2017.8079981.

Alexandru, A. et al. (2016) 'Healthcare, Big Data and Cloud Computing', WSEAS Transactions on Computer Research, 4, pp. 123-131.

Alhenaki, L. et al. (2019) 'A Survey on the Security of Cloud Computing', in. doi: 10.1109/cais.2019.8769497.

Appleford, G., \& Ramarao, S. (2019) 'Health Financing and Family Planning in the Context of Universal Health Care : Connecting the Discourse in Kenya'.

Barasa, E. W. et al. (2017) 'Recentralization within decentralization: County hospital autonomy under devolution in Kenya', PLoS ONE. doi: 10.1371/journal.pone.0182440.

Bhardwaj, T. and Sharma, S. C. (2018) 'Cloud-WBAN: An experimental framework for Cloud-enabled Wireless Body Area Network with efficient virtual resource utilization', Sustainable Computing: Informatics and Systems. doi: 10.1016/j.suscom.2018.08.008.

Bitta, M. A. et al. (2017) 'An overview of mental health care system in Kilifi, Kenya: Results from an initial assessment using the World Health Organization's Assessment Instrument for Mental Health Systems', International Journal of Mental Health Systems. doi: 10.1186/s13033-017-0135-5.

Bolognini, L. and Balboni, P. (2019) 'Iot and cloud computing: Specific security and data protection issues', in Internet of Things. doi: 10.1007/978-3-030-04984-3_4.

Boopathy, D. and Sundaresan, M. (2016) 'Secured cloud data storage - prototype trust model for public cloud storage', in Advances in Intelligent Systems and Computing. doi: 10.1007/978-981-10-0129-1_35.

Botta, A. et al. (2016) 'Integration of Cloud computing and Internet of Things: A survey', Future Generation Computer Systems. doi: 10.1016/j.future.2015.09.021.

Calabrese, B. and Cannataro, M. (2015) 'Cloud computing in healthcare and biomedicine', Scalable Computing. doi: $10.12694 /$ scpe.v16i1.1057.

Costa, G. et al. (2018) 'Automatic security verification of mobile app configurations', Future Generation Computer Systems. doi: 10.1016/j.future.2016.06.014.

Cruz, L. and Abreu, R. (2019) 'Catalog of energy patterns for mobile applications', Empirical Software Engineering. doi: 10.1007/s10664-019-09682-0.

Davis, E. C. et al. (2019) 'Examining healthcare systems: a market analysis for Kenya', European Journal of Training and Development. doi: 10.1108/EJTD-06-2016-0041.

Din, S. and Paul, A. (2019) 'Smart health monitoring and management system: Toward autonomous wearable sensing for Internet of Things using big data analytics', Future Generation Computer Systems. doi: 
Dohlman, L. et al. (2019) 'Global brain drain: How can the maslow theory of motivation improve our understanding of physician migration?', International Journal of Environmental Research and Public Health. doi: 10.3390/ijerph16071182.

Erdeniz, S. P. et al. (2018) 'Recommender systems for iot enabled m-health applications', in IFIP Advances in Information and Communication Technology. doi: 10.1007/978-3-319-92016-0_21.

Fielding, M., Odero, B. and Ochieng, C. (2016) 'From paper to data: taking medical health records into the future', Innovation cases study proposal for Nairobi Innovation Week 2016, p. 7.

Freiberg, M. A. T. et al. (2016) 'Chronic diseases in Nyeri, Kenya: a study of knowledge and perceptions', Healthcare in Low-resource Settings. doi: 10.4081/hls.2016.5669.

Gill, S. S. and Buyya, R. (2019) 'Sustainable Cloud Computing Realization for Different Applications: A Manifesto’, in. doi: 10.1007/978-3-319-93940-7_4.

Gupta, A., Chakraborty, C. and Gupta, B. (2019) 'Medical information processing using smartphone under IoT framework', in Studies in Systems, Decision and Control. doi: 10.1007/978-981-13-7399-2_12.

Halepoto, I. A. et al. (2018) 'Mobility management using the IP protocol', International Journal of Advanced Computer Science and Applications. doi: 10.14569/IJACSA.2018.090562.

Hamine, S. et al. (2015) 'Impact of mHealth chronic disease management on treatment adherence and patient outcomes: A systematic review’, Journal of Medical Internet Research. doi: 10.2196/jmir.3951.

Hettiarachchi, I. T. et al. (2019) 'Validation of Polar OH1 optical heart rate sensor for moderate and high intensity physical activities’, PLoS ONE. doi: 10.1371/journal.pone.0217288.

Hopia, H. et al. (2015) 'A patient as a self-manager of their personal data on health and disease with new technology - challenges for nursing education', Nurse Education Today. doi: 10.1016/j.nedt.2015.08.017.

Isemeck, C. S., Ngure, K. and Kariuki, J. (2019) 'Factors Influencing the Adoption of Electronic Health Records in Public Health Facilities in Kisumu County, Kenya', Journal of Health, Medicine and Nursing.

Kabia, E. et al. (2019) “"We are called the et cetera”: Experiences of the poor with health financing reforms that target them in Kenya', International Journal for Equity in Health. doi: 10.1186/s12939-019-1006-2.

Kimani, D. (2019) 'Are We Ready for a Data Bank? An Analysis of the Sufficiency of Kenya's Legal and Institutional Framework on Data Protection and Identity Theft', SSRN Electronic Journal. doi: $10.2139 /$ ssrn.3424673.

Kshetri, N. and Alcantara, L. L. (2016) 'Cloud Computing in Emerging Markets', in Encyclopedia of Cloud Computing. doi: 10.1002/9781118821930.ch54.

Kumar, Nabish et al. (2016) 'A GCM and GPS based approach to health service', in ACM International Conference Proceeding Series. doi: 10.1145/2905055.2905333.

Lam, F. et al. (2019) 'A retrospective mixed-methods evaluation of a national ORS and zinc scale-up program in Uganda between 2011 and 2016', Journal of global health. doi: 10.7189/jogh.09.010504.

Le-Tien, T. et al. (2018) 'Mobile and wireless sensor healthcare model based on cloud computing', in IFMBE Proceedings. doi: 10.1007/978-981-10-4361-1_99.

Lins, S., Schneider, S. and Sunyaev, A. (2018) 'Trust is Good, Control is Better: Creating Secure Clouds by Continuous Auditing', IEEE Transactions on Cloud Computing. doi: 10.1109/TCC.2016.2522411.

Magno, M. et al. (2017) 'DeepEmote: Towards multi-layer neural networks in a low power wearable multisensors bracelet', in Proceedings - 2017 7th International Workshop on Advances in Sensors and Interfaces, IWASI 2017. doi: 10.1109/IWASI.2017.7974208.

Manya, A., Nielsen, P. and Pundo, R. (2016) 'Cloud Computing as a Catalyst for Integrated Health Information Systems in Developing Countries', Association for Information Systems.

Martínez-Pérez, B., de la Torre-Díez, I. and López-Coronado, M. (2015) 'Privacy and Security in Mobile Health Apps: A Review and Recommendations', Journal of Medical Systems. doi: 10.1007/s10916-014-0181-3.

Metcalf, D. et al. (2016) 'Wearables and the internet of things for health: Wearable, interconnected devices promise more efficient and comprehensive health care', IEEE Pulse. doi: 10.1109/MPUL.2016.2592260.

mHealth Kenya Ltd (2015) Strategic Plan 2015-2020. Nairobi. Available at: https://mhealthkenya.org/wp- 
content/uploads/2017/03/mHealth-Kenya-Strategic-Plan.pdf.

Mills, E. J. and Lester, R. T. (2019) 'Mobile phone-enabled adherence in HIV/AIDS', The Lancet Digital Health. doi: 10.1016/s2589-7500(19)30008-1.

Munguti, S. N. (2018) 'Factors Influencing the Adoption of Cloud Computing in Software Development Companies in Kenya', International Academic Journal of Information Systems and Technology, 2(1), pp. $126-144$

Obadha, M. et al. (2019) 'Health care purchasing in Kenya: Experiences of health care providers with capitation and fee-for-service provider payment mechanisms', International Journal of Health Planning and Management. doi: 10.1002/hpm.2707.

Perlman, L. and Wechsler, M. (2019) 'Mobile Coverage and its Impact on Digital Financial Services', SSRN Electronic Journal. doi: 10.2139/ssrn.3370669.

Phillips, S. M. et al. (2018) 'Wearable Technology and Physical Activity in Chronic Disease: Opportunities and Challenges', American Journal of Preventive Medicine. doi: 10.1016/j.amepre.2017.08.015.

Plageras, A. P. et al. (2016) 'IoT-based surveillance system for ubiquitous healthcare', in IECON Proceedings (Industrial Electronics Conference). doi: 10.1109/IECON.2016.7793281.

Rahi, S. B., Bisui, S. and Misra, S. C. (2017) 'Identifying critical challenges in the adoption of cloud-based services', International Journal of Communication Systems. doi: 10.1002/dac.3261.

Salleh, N. A. et al. (2018) 'A systematic literature review of cloud computing adoption and impacts among small medium enterprises (SMEs)', in Proceedings - International Conference on Information and Communication Technology for the Muslim World 2018, ICT4M 2018. doi: 10.1109/ICT4M.2018.00058

Shah, G. H., Newell, B. and Whitworth, R. E. (2016) 'Health departments' engagement in emergency preparedness activities: The influence of health informatics capacity', International Journal of Health Policy and Management. doi: 10.15171/ijhpm.2016.48.

Slotwiner, D. J. et al. (2019) 'Transparent sharing of digital health data: A call to action', Heart Rhythm. doi: 10.1016/j.hrthm.2019.04.042.

Sony, P. and Sureshkumar, N. (2019) 'Concept-based electronic health record retrieval system in healthcare iot', in Advances in Intelligent Systems and Computing. doi: 10.1007/978-981-13-0617-4_17.

Taylor, V. F. et al. (2018) 'Robust Smartphone App Identification via Encrypted Network Traffic Analysis', IEEE Transactions on Information Forensics and Security. doi: 10.1109/TIFS.2017.2737970.

Thinakaran, K. et al. (2018) 'Developing a Privacy Compliance Scale for IoT Health Applications', Computer Science and Information Technology. doi: 10.13189/csit.2018.060402.

Toma, T., Masuda, Y. and Yamamoto, S. (2019) 'Vision paper for enabling digital healthcare applications in OHP2030', in Smart Innovation, Systems and Technologies. doi: 10.1007/978-3-319-92231-7_19.

Vazirani, A. A. et al. (2019) 'Implementing blockchains for efficient health care: Systematic review', Journal of Medical Internet Research. doi: 10.2196/12439.

Voigt, P. et al. (2017) 'Enforcement and Fines Under the GDPR', in The EU General Data Protection Regulation (GDPR). doi: 10.1007/978-3-319-57959-7_7. 